\title{
Correction to: The Use of Ammonia as a Fuel for Combustion Engines
}

Dong Han, Yusen Liu, and Zhen Huang

Correction to:

Chapter 10 in: G. Kalghatgi et al. (eds.), Engines and Fuels for Future Transport, Energy, Environment, and Sustainability, https://doi.org/10.1007/978-981-16-8717-4_10

In the original version of this chapter, the author mistakenly included the word "Gasoline" instead of "Hydrogen" in the abstract.

The correction chapter has been updated with the change. 